neither the fungus Mucor ramannianus nor the yeast Rhodotorula rubra will grow at all. If mixed inocula are used, both organisms grow perfectly. The reason for this surprising result is that the Mucor is unable to synthesize the pyrimidine moiety of aneurin but is able to synthesize the thiazole moiety, whereas Rhodotorula is able to synthesize the pyrimidine but not the thiazole. Thus the two in association act in a complementary manner and growth becomes possible.

In view of many such instances of a chemical background for symbiotic relations, it seems possible that the production of antibiotic substances may have some significance in explaining 'antibiotic relations', whether of the nature of the parasitism of fungi on higher plants or antagonism between fungi and other micro-organisms. Various plant juices have proved to be 'antibiotic'. Fragments of an orchid tuber suppress the growth of its mycorrhizal fungus; this may be related to the fact that the fungus is always restricted to definite areas of the growing root. These facts lead to the tentative suggestion that antibiosis may on occasion be an essential ingredient of symbiosis. Dr. Ramsbottom quoted the antagonism between Trichoderma viride and other fungi, with its possible connexion with gliotoxin and viridin production; the antagonism demonstrated by Van Luijk in 1939 between Penicillium expansum and Pythium spp. and its possible connexion with patulin production; the relation between the wilt-fungus Fusarium lycopersici and the tomato plant, in connexion with production of the wilt-producing polypeptide lycomarasmin. Caution is necessary in interpreting these facts ; in Dr. Ramsbottom's words "the balance [between organisms] is probably controlled to some extent by the ability of some organisms to produce antibiotics, though it would be teleological to suppose that they have a purposeful origin".

Dr. S. E. Michael wound up the discussion by considering the chemical structure of mould antibiotics in relation to the general metabolic chemistry of fungi. Systematic study of the metabolism of lower fungi by Raistrick and his colleagues has shown that moulds of one or of several species yield products which are elosely related variants of a particular type of structure. Examples that can be quoted are the toluquinone series (fumigatin and spinulosin); the anthraquinone series of mould pigments (helminthosporin, cynodontin, $\omega$-hydroxyemodin, catenarin and erythroglaucin); the tetronic acid series (carolic acid, dehydrocarolic acid, ascorbic acid and penicillic acid). These few examples-far from completeshow the remarkable variety of compounds found as metabolic products of moulds, and the tendency for these products to be variants of a smaller number of basic patterns of molecular structure.

A number of well-known antibiotics fall into one or other of these general classes. Those of the tetronic acid series are particularly interesting. Tetronic acid belongs to the important and widely distributed class of unsaturated $\gamma$-lactones. This unsaturated lactone structure has been found in a number of antibiotic substances, and it is of considerable interest to see how variations on this basic molecular structure affect biological activity. The whole carolic acid series and ascorbic acid are devoid of antibiotic activity. On the other hand, the antibiotics penicillic acid, patulin, vulpinic acid and protoanemonin (extracted from higher plants) are all unsaturated $\gamma$. lactones. If their structure is considered, it appears that activity is associated with an unsaturated lactone ring carrying at least one ethylenic or carbonyl radical outside the ring, and also in some cases a methoxy or other ether group attached to the unsaturated carbon atom of the ring. Where the unsaturated radical outside the ring is missing (dihydropenicillic acid, $\alpha$ - $\beta$-angelicalactone), or where the ether group has been replaced by a hydroxy-group (the carolic acid series), antibiotic activity is reduced or absent.

These examples show that many mould antibiotics can be recognized as variants or derivatives of structurally known metabolic products. Others, on the other hand, such as penicillin, appear at our present state of knowledge to possess chemical structures not hitherto recognized in natural products.

The wide field covered by this discussion makes it difficult for either a chemist or a biologist to appreciate the significance of all the points brought forward. Nevertheless, it is hoped that this short review will help to indicate the varied types of interest in antibiotics now being shown in different branches of science. It should also be explained that, in order to give a logical presentation of the material, the order in which the various contributions were given has been altered.

P. W. Brian

\section{CRACK DETECTION}

A TWO-DAY symposium on methods of crack detection was held under the auspices of the Industrial Radiology Group of the Institute of Physics during July 18-19 in the lecture theatre of the Electric Lamp Manufacturers' Association, Savoy Hill, London.

The chairman, Mr. W. J. Wiltshire, explained that the group has recently included lectures comparing other methods of non-destructive testing with radiological methods, and the symposium was an instance of this extension of activity.

A short address was given by Dr. W. Betteridge, who explained the importance of cracks in both metallic and non-metallic components, the various means by which the main types of crack are formed, and the principal methods of detection. The latter he divided into three groups : the essentially visual methods, including the ways of making a crack more apparent, such as etching, dyeing, magnetic ink indication, etc., and radiography, the visual examina. tion then being made on the film instead of on the actual component; the inferential methods such as the use of supersonics or of electrical resistivity, in which the presence of a crack is deduced from its disturbance of the normal lines of sound or current flow; and the mechanical methods, including proof loading, pressure testing, damping, etc.

Mr. C. Croxson dealt in detail with the application of radiography to the detection of cracks. He said that not many years ago a high-grade component containing a crack would have been immediately condemned; but nowadays repairs by excision and welding under radiographic supervision are often satisfactorily performed. A discouraging factor is the difficulty of assessing the mechanical significance of a crack by correlating radiographic results with practical experience. Penumbral unsharpness, due either to a broad focus tube or a small target-specimen distance, is not very detrimental and, within limits, may even lead to increased sensitivity; but graininess in the film is a great handicap, and the use of the finest grained film is recommended. The sensitivity of radiographic methods is higher than generally sup- 
posed, and cracks only a few ten-thousandths of an inch in width can be detected in steel plates up to half an inch in thickness if favourably oriented. The discernibility of a crack is related to the ratio of the width to the angle of inclination to the X-ray beam; but for shallow cracks, where the inclination does not noticeably change the appearance of the image, it depends on the cross-section of the crack. Mr. Croxson concluded with a number of illustrations of the application of radiography to the detection of cracks in castings, forgings and welds, and, in summarizing the usefulness of the method, said that, in his opinion, serious hot tears or cold cracks in castings could not escape detection by radiography, and the same applied to cracks in forgings provided a critical technique was used with an understanding of the probable location and direction of the cracks. In welds small cracks could escape detection, but these are usually of little practical significance.

Mr. P. Rowbotham surveyed the various visual, etching and magnetic methods of detection, and explained that for all these the best results could only be expected if the inspectors worked under optimum conditions of lighting. A trained inspector working under ideal conditions can achieve very high sensitivity. Magnifying lenses and low-power microscopes can be helpful, but the small field of view and the necessity for frequent refocusing are disadvantages. Their value is mainly in the assessment of a crack following its detection by a more sensitive method. Brief descriptions were given of several common methods of increasing the visibility of a crack, including etching, anodizing, the use of oil and chalk, fluorescent liquids, etc., all these depending on the absorption into the crack of a liquid which afterwards oozes out. Finally, the magnetic methods for ferromagnetic materials, in which the escape of flux at the crack is indicated by a detector fluid, were described. For all these methods the crack must break through the surface of the part, except that the magnetic method will reveal cracks which are only a small distance beneath the surface.

The use of electrical conductivity methods for non-magnetic metals was dealt with by Mr. A. M. Armour, who explained that while a crack will disturb the lines of current flow in a metal in the same way as the lines of magnetic force in a ferromagnetic material, the electrical method is much less sensitive than the magnetic method because of the lack of a sufficiently sensitive detector of the lines of current flow. Most success has been achieved by the use of scanning search coils coupled to an indicator to give a signal on passing the vicinity of a defect. The use of two balanced search coils sym. metrically placed with respect to a primary coil has enabled relatively uniform material such as sheet or bar to be examined successfully; but the method fails when a complicated component is to be dealt with because of the disturbances introduced by changes of section. Success has also been obtained in the search for diametrical cracks in large light-alloy billets by rotating the billet in a magnetic field and causing the eddy currents produced to react on two opposed search coils. A direct-current potential drop method has also proved of value for the detection of laminations in sheet, lack of adhesion in bearing liners, etc.

Supersonic methods were described by Mr. D. O. Sproule. The shadow detection method, in which the effect of a defect on the transmitted primary beam is observed, was considered to be of low sensitivity compared with echo methods, and the method in which the reflected beam interferes with the frequency-modulated primary beam was also described as of less importance than the echo method using pulse excitation. In the latter case the primary beam consists of pulses of sound at a frequency of a few megacycles per second produced by an oscillating quartz crystal, and the reflected energy is picked up by a similar crystal in the intervals between the primary pulses. The depth of the defect is determined by the time interval between the primary and reflected pulses ; but the character of the defect producing the echo is not readily determined, although, by frequency variation, defects of relatively large areas, such as cracks, can be differentiated from fine porosity. The application of the method to components of different shapes was described, and the sensitivity to such small defects as hair-line cracks in large steol billets was illustrated.

The last paper was by Mr. M. Baimler, who described the development of industrial radiology in Czechoslovakia. The general position is very similar to that in Great Britain ; but the advantage of using a long anode tube, which radiates in all directions in one plane and thus enables a circle of a large number of components to be radiographed at a single exposure, was stressed; the tube-film distance of about 12 feet gives sharp definition, and the increased exposure time is more than compensated for by the number of components dealt with at one time. An ingenious screening stand for dealing with castings weighing up to about 3 cwt. was described.

In the discussion which followed, Mr. A. G. Warren made a plea for the use of the term ultrasonic to describe high-frequency sound, supersonic being used in connexion with speeds above that of sound. The need for a detector for electrical conductivity methods was met in the case of high-frequency currents by sparking across the crack, which was easily visible. $\mathrm{He}$ said that cracks at angles above $45^{\circ}$ to the direction of the lines of force could easily be detected by the magnetic ink method if the flux density were high enough. Mr. Rowbotham agreed, but found it preferable to control the flux. density so that the limit of sensitivity was at about $45^{\circ}$. Mr. Armour described a combined radiographic and magnetic method, the exposed and processed film forming the base on which the magnetic record is deposited by a special detector ink. Mr. H. Capper said that fatigue cracks are frequently missed by radiography, and complementary methods should always be applied. Permanent magnets are found useful for magnetic crack detection. Eddy current methods have been found by Mr. E. G. Stanford to be of value for checking consistency of products for dimensions, composition, temper, etc., and the changes introduced by such variables make crack detection ambiguous. Mr. D. J. Jones described the difficulty of finding longitudinal cracks in sealing wires for the lamp industry; visual examination at a magnification of 10-20 was found best. Mr. W. C. Heselwood had found the shadow ultrasonic method preferable to the potential drop method for the detection of laminations in steel plate. In answer to a question by Mr. A. Maskery on the possibility of using a single exploring head for ultrasonic work, Mr. Sproule described a method of using two balanced heads, one on a standard sample and the second exploring the test sample, defects then being indicated by a disturbance of the balance; but he thought that a single head generally gave less information than the separate transmitter and detector. 